\title{
Negative regulation of receptor tyrosine kinases: unexpected links to c-Cbl and receptor ubiquitylation
}

\author{
Chanan RUBIN, Gal GUR, Yosef YARDEN* \\ Department of Biological Regulation, The Weizmann Institute of Science, Rehovot 76100, Israel
}

\begin{abstract}
Intracellular signals mediated by the family of receptor tyrosine kinases play pivotal roles in morphogenesis, cell fate determination and pathogenesis. Precise control of signal amplitude and duration is critical for the fidelity and robustness of these processes. Activation of receptor tyrosine kinases by their cognate growth factors not only leads to propagation of the signal through various biochemical cascades, but also sets in motion multiple attenuation mechanisms that ultimately terminate the active state. Early attenuators pre-exist prior to receptor activation and they act to limit signal propagation. Subsequently, late attenuators, such as Lrig and Sprouty, are transcriptionally induced and further act to dampen the signal. Central to the process of signaling attenuation is the role of the E3 ubiquitin ligase c-Cbl. While Cblmediated processes of receptor ubiquitylation and endocytosis are relatively well understood, the links of $\mathrm{Cbl}$ to other negative regulators are just now beginning to be appreciated. Here we review some emerging interfaces between $\mathrm{Cbl}$ and the transcriptionally induced negative regulators Lrig and Sprouty.
\end{abstract}

Keywords: endocytosis, growth factor, oncogene, signal transduction, ubiquitin, tyrosine kinase.

\section{INTRODUCTION}

Receptor tyrosine kinases (RTKs) have evolved as primary mediators of cell-to-cell communication in multicellular organisms. These membrane-spanning proteins mediate a variety of cellular responses ranging from cell migration to survival, cell proliferation and differentiation. Ligand binding to the cognate receptor triggers receptor dimerization and activation of the kinase domain, rendering the receptor catalytically active. Receptor trans- and auto-phosphorylation on tyrosine residues located within the cytoplasmic tail create docking sites for proteins containing phosphotyrosine-binding modules. In turn, the recruited proteins initiate various signaling cascades [1]. Tuning of signaling amplitude and duration is crucial for induction of the correct physiological outcome [2]. For example, the patterning of the Drosophila embryonic ventral ectoderm relies on different levels of epidermal growth factor receptor (EGFR) signaling for the induction of various cell fates [reviewed in [3]]. In this vein, ventral midline cells provide the source for Spitz,

*Correspondence: Yosef YARDEN

Tel: +972-8-9343974; Fax: +972-8-9342488;

E-mail: yosef.yarden@weizmann.ac.il a soluble ligand for the Drosophila EGF-receptor (DER). Cells in close proximity to midline cells experience high levels of DER activation and express a specific set of markers, including orthodenticle. More distal cells exhibit lower DER activation and express other markers such as fasciclin III.

It is becoming apparent that a large set of inhibitory proteins act to attenuate the signal emanating from activated receptors (Tab. 1). These negative regulators can either exist prior to receptor activation or they are newly synthesized following signaling initiation (see Fig. 1). Indeed, receptor activation not only instigates multiple positively acting pathways, such as the Ras-mitogen-activated protein kinase (MAPK); and the phosphatidylinositol 3kinase (PI3K)-Akt cascades, but also sets in motion mechanisms that will ultimately terminate signaling. The induction of negative feedback loops has been well characterized in insects. Examples of genes that are transcriptionally induced following receptor activation include the intracellular membrane-bound protein Sprouty [4], the membrane spanning Kekkon proteins [5] and the antagonistic ligand of the insect EGFR, namely Argos [6]. Similarly, mammalian RTK attenuators such as the pan-ErbB inhibitor RALT/Mig-6 [7] and the fibroblasts-derived growth factor receptor (FGFR) inhibitor, Sef [8], are also newly synthe- 


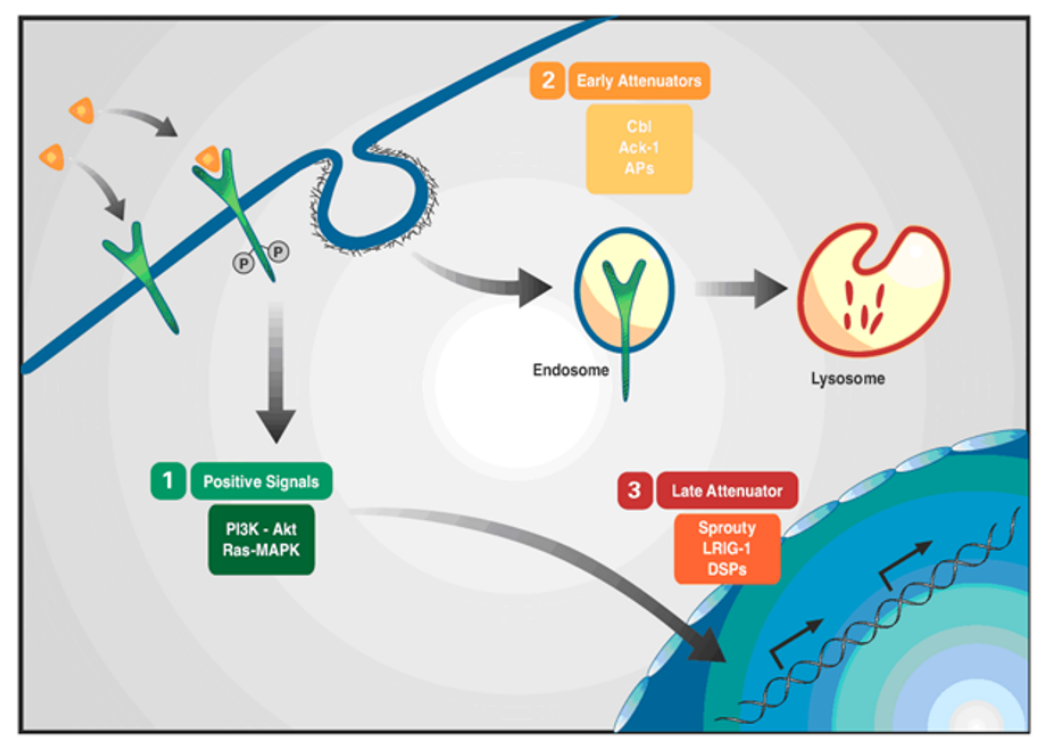

Fig. 1 Activation-dependent mechanisms of signal attenuation. Growth factor binding to a receptor tyrosine kinase induces receptor auto-phosphorylation, followed by simultaneous activation of multiple positive signaling pathways. Cbl-mediated receptor ubiquitylation marks the onset of attenuation, starting with pre-existing molecules involved in endocytosis and cytoskeleton rearrangement (early attenuators), and culminating in transcription-dependent negative regulatory pathways (late attenuators). The list of up-regulated genes includes those encoding MAPK-specific phosphatases, various adaptors, transcription factors and secondary growth factors. DSPs, dual-specificity phosphatases; APs, clathrin-associated proteins.

Tab. 1 Negative regulators of RTKs and their modes of action

\begin{tabular}{llll}
\hline Protein name & Domain structure & Inhibitory targets & Mechanism of action \\
\hline Ack1 & TK, SH3, CRIB, UBA & EGFR & Unknown \\
Argos & EGF & DER & Ligand sequestration \\
c-Cbl & SH2, RF, UBA & Several RTKs & Mediates receptor ubiquitylation \\
Echinoid & Ig-like, FN3, TM & EGFR & Unknown [49] \\
Kekkon & LRR, Ig-like, TM & DER & Inhibits growth factor binding \\
LRIG1 & LRR, Ig-like, TM & ErbB family members & Enhances receptor ubiquitylation \\
Nedd4 & C2, WW, HECT & IGF1R, VEGFR & Mediates receptor ubiquitylation [50,51] \\
Sef & None identified & Multiple RTKs & Retains Erk in the cytoplasm [8,52] \\
Senseless & Zinc fingers & DER & Represses pointed- mediated transcription [53] \\
Socs 1 and 3 & SH2, SOCS box & Insulin and cytokine receptors & Mediate IRS1/2 \\
& & & ubiquitylation and block access of substrates [54] \\
Spred & EVH-1, CRD & RTKs & Inhibits Ras-mediated Raf activation [55] \\
Sprouty & CRD & Several RTKs & Unknown \\
Ralt/Mig6 & None identified & EGFR, ErbB-2 & Unknown [7] \\
\hline
\end{tabular}

sized in response to growth factors.

\section{REGULATION OF RECEPTOR ENDOCYTOSIS}

Of the negative regulators, which are independent of new transcription, the E3 ubiquitin ligase c-Cbl/Sli-1 is relatively well understood. Screens for suppressors of hypomorphic mutations of EGFR/LET-23 in C. elegans identified SLI-1 as a negative regulator of the EGFR signaling pathway [9]. In line with genetic evidence indicating that SLI-1 acts downstream to the receptor and upstream of Ras, c-Cbl, a mammalian ortholog of SLI-1, was shown to be rapidly phosphorylated and to complex 


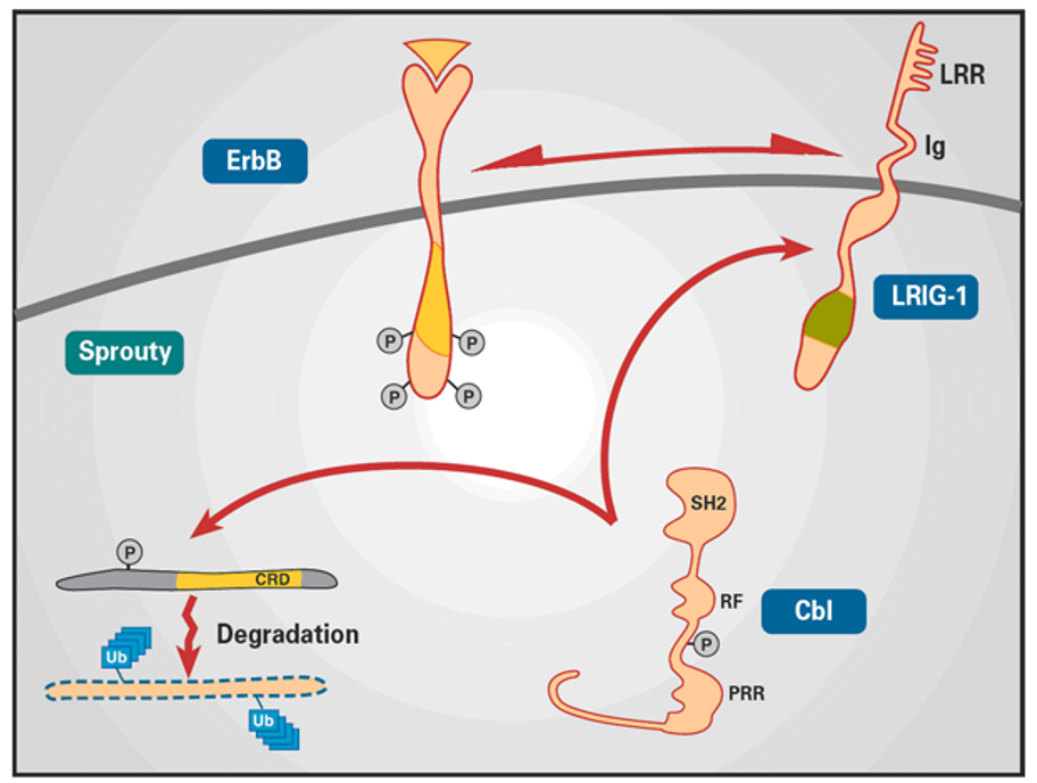

Fig. 2 Modulators of ErbB signaling. The domain structures of LRIG-1, Cbl and Sprouty proteins are schematically presented. Arrows indicate direct or indirect interactions. Thus, the extracellular domains of ErbB and LRIG proteins physically interact in a ligand-independent manner, and ErbB increases tyrosine phosphorylation of Sprouty. Cbl proteins bind in a phosphorylationdependent manner to ErbB and Sprouty proteins, but the interaction with LRIG-1 is phosphorylation-independent. All three targets of $\mathrm{Cbl}$ undergo ubiquitylation, which dictates degradation by lysosomes or proteasomes. CRD, cysteine-rich domain; Ig, immunoglobulin-like domain; LRD, leucine-rich region; PRR, proline-rich domain; RF, RING finger.

with the EGFR following EGF stimulation [10]. The domain structure of c-Cbl consists of a tyrosine kinase binding (TKB) domain through which it binds tyrosinephosphorylated targets, a RING finger domain, which binds E2-conjugating enzymes, and a C-terminal ubiquitin-associated (UBA) domain. Additional studies demonstrated that c-Cbl facilitates ligand-induced ubiquitylation of the EGFR $[11,12]$, as well as several other RTKs (reviewed in [13]) by means of RING finger-dependant recruitment of E2 ubiquitin-conjugating enzymes to the receptor's vicinity. Receptor ubiquitylation results in accelerated removal from the cell surface and to subsequent receptor degradation in the lysosomal compartment, thereby terminating RTK signaling.

Recent studies have linked RTK ubiquitylation to the process of receptor endocytosis [14]. This phenomenon entails the internalization of the receptor from the plasma membrane and its routing through several intracellular compartments. Multiple sorting steps, along the endocytotic course, ultimately determine whether the receptor will be destined for degradation or recycle back to the cell surface. Although receptor degradation is a possible consequence of endocytosis, this process cannot be solely considered as a signal termination mechanism. Emerging data indicate that the specificity, kinetics and magnitude of receptor's responses may be regulated by the locationof the activated receptor in the endocytotic pathway [15].

Ligand binding initiates receptor auto-phosphorylation, which is followed by the recruitment of the Cbl TKB domain to a specific phosphotyrosine, such as tyrosine 1045 of the EGFR [11]. This proximity facilitates Cbl-mediated receptor mono-ubiquitylation [16,17], which commences at the plasma membrane $[18,19]$. It is believed that monoubiquitylation of the receptor on multiple lysine residues robustly generates docking sites for endocytotic adaptor proteins possessing ubiquitin-binding domains. Adaptors, such as Eps15, may recruit receptors to clathrin-coated pits as they comprise both ubiquitin interacting motifs (UIMs) [20] and DPF motifs that couple to clathrin adaptors [AP2; [21]]. Recruited receptors are thus linked to AP2 complexes that drive the assembly of clathrin-coated vesicles (CCVs). The CCVs shed clathrin and fuse with internal vesicles to form early endosomes that proceed along the endocytic pathway to the multi-vesicular body (MVB). At the MVB, endocytotic adaptors sort receptors for destruction in the lysosome or for recycling vesicles, 
which eventually fuse with the plasma membrane [22]. This final sorting step is also regulated by the E3 ligase activity of Cbl; sustained ubiquitylation of the EGFR in endosomes was shown to be necessary for the sorting to lysosomal compartments [18], implying that Cbl-mediated ubiquitylation serves as a switch shunting activated receptors for degradation

The primordial form of $\mathrm{Cbl}$ appeared relatively early during evolution in nematodes such as $C$. elegans. Two negative regulators of RTKs, namely Sprouty and Kekkon, which have subsequently evolved in insects, retain complex relationships with $\mathrm{Cbl}$ both in insects and in mammals [2326]. In addition, the association of these inhibitors with $\mathrm{Cbl}$ leads to the ubiquitylation and degradation of Sprouty and LRIG in the proteasome, which subsequently limits their inhibitory capacity (see Fig. 2). Hence, it seems that evolution has coupled its more recent inventions to the more ancient ones, designating $\mathrm{Cbl}$ not only as a regulator of RTKs but also as master regulator of secondary regulators, as described below.

\section{SPROUTY PROTEINS: DUAL REGULATORS OF RTK SIGNALING}

Sprouty was originally identified in Drosophila as an antagonist of Breathless, the insect equivalent of the fibroblast growth factor receptor (FGFR; [27]). Additional studies demonstrated that Sprouty also inhibits the Drosophila EGFR (DER), as well as other RTKs [28]. Four orthologs of the Drosophila sprouty (dspry) have been identified in mammals. Of these, spry2 exhibits the highest level of homology to the ancestral gene. Functionally, human Spry2 has retained its regulatory role, impeding activation of the MAPK by some, but not all RTKs. The negatively regulated mammalian RTKs include the receptors for FGF, the vascular endothelial growth factor and the hepatocyte growth factor $[29,30]$. In contrast, the effect of Spry2 on signaling downstream to the epidermal growth factor receptor (EGFR) seems more complex [31]. Likewise, it is still unclear at which level Sprouty proteins regulate MAPK signaling. Spry2 appears to physically interact with multiple components of the Ras-MAPK pathway, including Grb2, FRS2, Raf1 the Ras GTPase activating protein (Ras-GAP; [32]) and c-Cbl [33]. Several regulatory mechanisms have been proposed, including inhibition at the levels of Grb2 [34], GAP [4], or the Raf1 kinase [35,36]. In contrast, the interactions with c-Cbl may positively impact on EGFR signaling [26,37].

In addition to its role as a regulator of RTK signaling, Spry2 itself is subject to tight regulation by RTKs on several levels. First, the activation of RTKs triggers an induction in spry2 transcription in epithelial cells [35]. Regulation of the mature protein is mediated by ligand- induced phosphorylation of Spry2 on a tyrosine residue located at position $55[24,26,34,38]$. This evolutionarily conserved tyrosine is essential for the inhibitory activity of Spry2 [35], although the exact mechanism remains unknown. In addition to its role as an operational switch, phosphorylation of tyrosine 55 creates a docking site for the E3 ubiquitin ligase c-Cbl. Following RTK activation, c-Cbl binds phosphorylated Spry2 through its TKB domain. This association results in the ubiquitylation and proteasomal degradation of active Spry2, thus limiting its inhibitory effects $[24,26]$. On the other hand, the association between Sprouty and c-Cbl may sequester the E3 ligase in a way that prevents ubiquitylation of target proteins. In summary, the interaction between Sprouty and Cbl emerges as a focal point not only in Sprouty's role in restraining RTK signals but also in the life cycle of Sprouty proteins themselves. We speculate that Cbl plays a dual role in this web; along with ubiquitylating Sproutyassociated proteins and sorting them for lysosomal or proteasomal degradation, Cbl's function as a multivalent adaptor capable of engaging more than 50 different proteins [39] may underlie the ability of Sprouty to interfere with signaling pathways.

\section{LRIG1 AS A NEGATIVE REGULATOR OF MAM- MALIAN RTKS}

Shortly following the identification of Sprouty as an inducible inhibitor of FGF-signaling, another feedback regulator was shown to inhibit the activity of DER during oogenesis [40]. This gene, named kekkon-1, encodes a transmembrane protein that physically binds to and directly inhibits EGFR molecules [41]. The six leucine-rich repeats (LRRs) of Kekkon-1 are necessary for recognition of EGFR, and for consequent inhibition of activation by growth factors [41,42]. The multiple Kekkon proteins of insects have no clear orthologs in mammals [43]. On the other hand, a clear ortholog of mammalian LRIG proteins exists in flies and nematodes. Nevertheless, the three mammalian LRIG genes share domain organization with Kekkons [44-47]. The extracellular regions of both the murine Lrig1/ Lig-1 [47] and the human LRIG1 [46] share 15 LRRs followed by three Ig domains. Interestingly, disruption of the LRIGI gene in mice resulted in fertile animals that developed defects in skin [48], a major site of EGFR action.

Similar to kekkon-1 and to other ligand-dependent negative feedback regulators of ErbB signaling, Lrig1 is transcriptionally up-regulated upon EGF stimulation [23]. The structural similarity of LRIG1 to Kekkons predicted that LRIG1 would interact with and restrict ErbB signaling in mammals. Indeed, LRIG1 localizes to the basolateral surface of epithelial cells, the site of ErbB function, and physically interacts with all members of the ErbB family $[23,25]$. 
This recognition involves both LRIG1's and the receptor's ectodomains and it requires no stimulation by the respective ErbB ligand. However, whereas only the LRRs of Kekkon-1 are necessary for recognition of the Drosophila EGFR ectodomain [41,42], the LRRs and the immunoglobulin- (Ig-) like domains of LRIG1 are each sufficient for receptor binding [23].

Ligand-induced up-regulation of LRIG-1 expression shortens the half-life of ErbB-1/EGFR, due to enhancement of ligand-induced receptor ubiquitylation and an associated sorting of ligand-activated receptors to intracellular degradation, thus restricting growth factor signaling [23]. Conclusions derived from both in vivo and in vitro assays suggest that the N-terminal half of $\mathrm{c}-\mathrm{Cbl}$ directly binds to the juxtamembrane region of LRIG1 [23]. By recruiting $\mathrm{c}-\mathrm{Cbl}$ to the vicinity of EGFR, both LRIG1 and the receptor undergo ubiquitylation and subsequent degradation [23,25]. Remarkably, Kekkon1-EGFR interaction inhibits EGFR signaling in an apparently different mechanism, which involves inhibition of growth factor binding, receptor auto-phosphorylation and MAPK activation in response to EGF [41]. In summary, both Drosophila's Kekkon proteins and mammalian LRIG family members are induced upon receptor activation and in both cases receptor signaling is subsequently blocked. However, LRIG and Kekkon proteins have no common genetic origin and their modes of action differ, implying evolutionary convergence of negative feedback mechanisms.

\section{PERSPECTIVES}

The growing interest in negative regulation of RTK signaling has led to the identification of multiple proteins involved in the process of signal attenuation. Although in many cases the mechanism of action has proven elusive, the overall picture is of regulation at numerous levels, including the level of the ligand, the receptor, the downstream signaling components and the respective target transcription factors (Tab. 1). Of these mechanisms, receptor downregulation by means of endocytosis emerges as a major player in signaling attenuation. Central to this process is the role of the $\mathrm{E} 3$ ligase $\mathrm{Cbl}$, which directs ligand-induced receptor ubiquitylation and subsequent degradation. Interestingly, additional negative regulators such as Sprouty and LRIG, which evolved later in the course of evolution, fine-tune $\mathrm{Cbl}$ activity and are themselves subjected to Cbl-mediated regulation. Unlike Cbl, both Sprouty and LRIG are transcriptionally induced upon receptor stimulation, thus participating in a large-scale negative feedback program. Future studies will help untangling the underlying genetic program and uncovering the many layers of receptor regulation.

\section{REFERENCES}

1 Blume-Jensen P, Hunter T. Oncogenic kinase signaling. Nature 2001; 411:355-65.

2 Marshall CJ. Specificity of receptor tyrosine kinase signaling: transient versus sustained extracellular signal-regulated kinase activation. Cell 1995; 80:179-85.

3 Shilo BZ. Signaling by the Drosophila epidermal growth factor receptor pathway during development. Exp Cell Res 2003; 284: 140-9.

4 Casci T, Vinos J, Freeman M. Sprouty, an intracellular inhibitor of Ras signaling. Cell 1999; 96:655-65.

5 Musacchio M, Perrimon N. The Drosophila kekkon genes: novel members of both the leucine-rich repeat and immunoglobulin superfamilies expressed in the CNS. Dev Biol 1996; 178: 63-76.

6 Golembo M, Schweitzer R, Freeman M, et al. Argos transcription is induced by the Drosophila EGF receptor pathway to form an inhibitory feedback loop. Development 1996; 122:22330.

7 Fiorini M, Ballaro C, Sala G, et al. Expression of RALT, a feedback inhibitor of ErbB receptors, is subjected to an integrated transcriptional and post-translational control. Oncogene 2002; 21:6530-9.

8 Tsang M, Dawid IB. Promotion and attenuation of FGF signaling through the Ras-MAPK pathway. Sci STKE 2004; 6:pe17.

9 Jongeward GD, Clandinin TR, Sternberg PW. sli-1, a negative regulator of let-23-mediated signaling in C. elegans. Genetics 1995; 139:1553-66.

10 Bowtell DD, Langdon WY. The protein product of the c-cbl oncogene rapidly complexes with the EGF receptor and is tyrosine phosphorylated following EGF stimulation. Oncogene 1995; 11:1561-7.

11 Levkowitz G, Waterman H, Ettenberg SA, et al. Ubiquitin ligase activity and tyrosine phosphorylation underlie suppression of growth factor signaling by c-Cb1/Sli-1. Mol Cell 1999; 4:102940.

12 Levkowitz G, Waterman H, Zamir E, et al. c-Cbl/Sli-1 regulates endocytic sorting and ubiquitination of the epidermal growth factor receptor. Genes Dev 1998; 12:3663-74.

13 Thien CB, Langdon WY. Cbl: many adaptations to regulate protein tyrosine kinases. Nat Rev Mol Cell Biol 2001; 2: 294-307.

14 Marmor MD, Yarden Y. Role of protein ubiquitylation in regulating endocytosis of receptor tyrosine kinases. Oncogene 2004; 23:2057-70.

15 Burke P, Schooler K, Wiley HS. Regulation of epidermal growth factor receptor signaling by endocytosis and intracellular trafficking. Mol Biol Cell 2001; 12:1897-910.

16 Haglund K, Sigismund S, Polo S, et al. Multiple monoubiquitination of RTKs is sufficient for their endocytosis and degradation. Nat Cell Biol 2003; 5:461-6.

17 Mosesson Y, Shtiegman K, Katz M, et al. Endocytosis of receptor tyrosine kinases is driven by monoubiquitylation, not polyubiquitylation. J Biol Chem 2003; 278:21323-6.

18 Longva KE, Blystad FD, Stang E, et al. Ubiquitination and proteasomal activity is required for transport of the EGF receptor to inner membranes of multivesicular bodies. J Cell Biol 2002; 156:843-54.

19 Stang E, Johannessen LE, Knardal SL, et al. Polyubiquitination of the epidermal growth factor receptor occurs at the plasma 
membrane upon ligand-induced activation. J Biol Chem 2000; 275:13940-7.

20 Polo S, Sigismund S, Faretta M, et al. A single motif responsible for ubiquitin recognition and monoubiquitination in endocytic proteins. Nature 2002; 416:451-5.

21 Salcini AE, Confalonieri S, Doria M, et al. Binding specificity and in vivo targets of the EH domain, a novel protein-protein interaction module. Genes Dev 1997; 11:2239-49.

22 Katzmann DJ, Odorizzi G, Emr SD. Receptor downregulation and multivesicular-body sorting. Nat Rev Mol Cell Biol 2002; 3: 893-905.

23 Gur G, Rubin C, Katz M, et al. LRIG1 restricts growth factor signaling by enhancing receptor ubiquitylation and degradation. EMBO J 2004; 23:3270-81.

24 Hall AB, Jura N, DaSilva J, et al. hSpry2 Is Targeted to the Ubiquitin-Dependent Proteasome Pathway by c-Cbl. Curr Biol 2003; 13:308-14.

25 Laederich MB, Funes-Duran M, Yen L, et al. The leucine-rich repeat protein LRIG1 is a negative regulator of ErbB family receptor tyrosine kinases. J Bil Chem 2004; 279:47050-6.

26 Rubin C, Litvak V, Medvedovsky H, et al. Sprouty fine-tunes EGF signaling through interlinked positive and negative feedback loops. Curr Biol 2003; 13:297-307.

27 Hacohen N, Kramer S, Sutherland D, et al. sprouty encodes a novel antagonist of FGF signaling that patterns apical branching of the Drosophila airways. Cell 1998; 92:253-63.

28 Reich A, Sapir A, Shilo B. Sprouty is a general inhibitor of receptor tyrosine kinase signaling. Development 1999; 126:4139-47.

29 Impagnatiello MA, Weitzer S, Gannon G, et al. Mammalian sprouty-1 and -2 are membrane-anchored phosphoprotein inhibitors of growth factor signaling in endothelial cells. J Cell Biol 2001; 152:1087-98.

30 Lee CC, Putnam AJ, Miranti CK, et al. Overexpression of sprouty 2 inhibits $\mathrm{HGF} / \mathrm{SF}-$ mediated cell growth, invasion, migration, and cytokinesis. Oncogene 2004; 23:5193-202.

31 Egan JE, Hall AB, Yatsula BA, et al. The bimodal regulation of epidermal growth factor signaling by human Sprouty proteins. Proc Natl Acad Sci U S A 2002; 99:6041-6.

32 Tefft D, Lee M, Smith S, et al. mSprouty2 inhibits FGF10activated MAP kinase by differentially binding to upstream target proteins. Am J Physiol Lung Cell Mol Physiol 2002; 283: L700-6.

33 Wong ES, Lim J, Low BC, et al. Evidence for direct interaction between Sprouty and Cbl. J Biol Chem 2001; 276:5866-75.

34 Hanafusa H, Toril S, Yasunaga T, et al. Sprouty1 and Sprouty2 provide a control mechanism for the Ras/MAPK signaling pathway. Nat Cell Biol 2002; 4:850-8.

35 Sasaki A, Taketomi T, Wakioka T, et al. Identification of a dominant negative mutant of Sprouty that potentiates fibroblast growth factor- but not epidermal growth factor-induced ERK activation. J Biol Chem 2001; 276:36804-8.

36 Yusoff P, Lao DH, Ong SH, et al. Sprouty2 inhibits the Ras/ MAP kinase pathway by inhibiting the activation of Raf. J Biol Chem 2002; 277:3195-201.

37 Wong ES, Fong CW, Lim J, et al. Sprouty2 attenuates epidermal growth factor receptor ubiquitylation and endocytosis, and consequently enhances Ras/ERK signaling. Embo J 2002; 21:4796808.
38 Fong CW, Leong HF, Wong ES, et al. Tyrosine phosphorylation of Sprouty2 enhances its interaction with c-Cbl and is crucial for its function. J Biol Chem 2003; 278:33456-64.

39 Tsygankov AY, Teckchandani AM, Feshchenko EA, et al. Beyond the RING: CBL proteins as multivalent adapters. Oncogene 2001; 20:6382-402.

40 Ghiglione C, Carraway KL, Amundadottir LT, et al. The transmembrane molecule kekkon 1 acts in a feedback loop to negatively regulate the activity of the Drosophila EGF receptor during oogenesis. Cell 1999; 96:847-56.

41 Ghiglione C, Amundadottir L, Andresdottir M, et al. Mechanism of inhibition of the Drosophila and mammalian EGF receptors by the transmembrane protein Kekkon 1. Development 2003; 130:4483-93.

42 Alvarado D, Rice AH, Duffy JB. Bipartite inhibition of Drosophila epidermal growth factor receptor by the extracellular and transmembrane domains of Kekkon1. Genetics 2004; 167:187202.

43 MacLaren CM, Evans TA, Alvarado D, et al. Comparative analysis of the Kekkon molecules, related members of the LIG superfamily. Dev Genes Evol 2004; 214:360-6.

44 Guo D, Holmlund C, Henriksson R, et al. The LRIG gene family has three vertebrate paralogs widely expressed in human and mouse tissues, and a homolg in Ascidiacea. Genomics 2004; 84: 157-65.

45 Holmlund C, Nilsson J, Guo D, et al. Characterization and tissuespecific expression of human LRIG2. Gene 2004; 332:35-43.

46 Nilsson J, Vallbo C, Guo D, et al. Cloning, characterization, and expression of human LIG1. Biochem Biophys Res Commun 2001; 284:1155-61.

47 Suzuki Y, Sato N, Tohyama M, et al. cDNA cloning of a novel membrane glycoprotein that is expressed specifically in glial cells in the mouse brain. LIG-1, a protein with leucine-rich repeats and immunoglobulin-like domains. J Biol Chem 1996; 271:225227.

48 Suzuki Y, Miura H, Tanemura A, et al. Targeted disruption of LIG-1 gene results in psoriasiform epidermal hyperplasia. FEBS Lett 2002; 521:67-71.

49 Bai J, Chiu W, Wang J, et al. The cell adhesion molecule Echinoid defines a new pathway that antagonizes the Drosophila EGF receptor signaling pathway. Development 2001; 128:591-601.

50 Murdaca J, Treins C, Monthouel-Kartmann MN, et al. Grb10 prevents Nedd4-mediated vascular endothelial growth factor receptor-2 degradation. J Biol chem 2004; 279:26754-61.

51 Vecchione A, Marchese A, Henry P, et al. The Grb10/Nedd4 complex regulates ligand-induced ubiquitination and stability of the insulin-like growth factor I receptor. Mol Cell Biol 2003; 23: 3363-72.

52 Torii S, Kusakabe M, Yamamoto T, et al. Sef is a spatial regulator for Ras/MAP kinase signaling. Dev Cell 2004; 7:33-41.

53 Frankfort BJ, Mardon G. Senseless represses nuclear transduction of Egfr pathway activation. Development 2004; 131:56370 .

54 Jiang J, Ballinger CA, Wu Y, et al. CHIP is a U-box-dependent E3 ubiquitin ligase: identification of $\mathrm{Hsc} 70$ as a target for ubiquitylation. J Biol Chem 2001; 276:42938-44.

55 Wakioka T, Sasaki A, Kato R, et al. Spred is a Sprouty-related suppressor of Ras signaling. Nature 2001; 412:647-51. 